\title{
Ubiquitous Surveillance Notional Architecture for System- Wide DAA Capabilities in the NAS
}

\author{
Chris A. Wargo \\ Mosaic ATM, Inc. \\ 540 Fort Evans Road N. E., Suite 300 \\ Leesburg, VA 21076 \\ 443-994-6137 \\ cwargo@mosaicatm.com \\ George Hunter \\ Mosaic ATM, Inc. \\ 540 Fort Evans Road N. E., Suite 300 \\ Leesburg, VA 21076 \\ 571-246-2531 \\ ghunter@mosaicatm.com \\ Terry Blumer \\ Aviation Management Associates, Inc. \\ 1325 G St., NW, Suite 500, \\ Washington, DC 20001 \\ 703-519-9923 \\ tblumer@avmgt.com \\ Pete Carros \\ Mosaic ATM, Inc. \\ 540 Fort Evans Road N. E., Suite 300 \\ Leesburg, VA 21076 \\ 570-854-5083 \\ pcarros@mosaicatm.com
}

\author{
Jason Glaneuski \\ Volpe National Transportation \\ Systems Center \\ 55 Broadway \\ Cambridge, MA 02142 \\ 617-599-3769 \\ Jason.Glaneuski@dot.gov \\ John DiFelici \\ Mosaic ATM, Inc. \\ 540 Fort Evans Road N. E., Suite 300 \\ Leesburg, VA 21076 \\ 571-246-2531 \\ jdifelici@mosaicatm.com \\ Dylan Hasson \\ Volpe National Transportation \\ Systems Center \\ 55 Broadway \\ Cambridge, MA 02142 \\ 617-494-2986 \\ Dylan.Hasson@dot.gov \\ Robert J. Kerczewski \\ NASA Glenn Research Center \\ 21000 Brookpark Rd \\ Cleveland, OH 44135 \\ $+12164333434$ \\ rkerczewski@nasa.gov
}

\begin{abstract}
There is an increasing demand for access to the national airspace system (NAS) by new entrants such as unmanned aircraft systems (UASs) and space vehicles. The need is driving the research into the development of a ubiquitous surveillance framework. A framework where all means of aircraft position tracking systems, both cooperative and noncooperative, would be correlated and made available to all NAS users. The architecture of an envisioned surveillance system was the focus of a recent Volpe National Transportation Systems Center research activity. In this work, the term "Framework" is used to characterize an operational environment that forms the context for future UAS operations. Within this framework, the use of all existing and future surveillance technologies is envisioned. Included would be all airspace locations; including coverage for airspace not under surveillance today. While these surveillance means and methods of air traffic control may currently constrain new entrant operations, they also provide the opportunity for the plethora of enabling technological capabilities with associated policies and procedures that can result in the safe, orderly and efficient operation. The focus of this paper is to survey and identify surveillance technologies to support the integration of new entrants in the NAS, and how those technologies can be aggregated.
\end{abstract}

\section{TABLE OF ConTEnTS}

1. INTRODUCTION ........................................................

2. FUTURE SURVEILLANCE NEEDS .............................2

3. SOURCES OF SURVEILLANCE ...............................4

4. ARCHITECTURAL ALTERNATIVES ........................9

5. CONCLUSIONS ...................................................12

ACKNOWLEDGEMENTS .............................................12

REFERENCES ..........................................................12

BIOGRAPHY .................................................................12

\section{INTRODUCTION}

The two chief surveillance targets in the NAS are vehicles and meteorological phenomena. For over half a century, these targets have been surveilled by surveillance technologies, systems, and infrastructure, which have been steadily 
increasing in capability and diversity. There has also been a growing diversity, particularly in recent years, of vehicles types in the NAS. Successfully integrating these new entrants into the NAS is an on-going challenge, and one requirement is to meet their surveillance needs.

This paper first examines the new entrants, and a sampling of their surveillance needs. We next survey the wide range of surveillance assets in the NAS, including both legacy and emerging systems and technologies. We then examine how these surveillance assets can meet the growing surveillance needs, and explore possible system architectures.

\section{Future SuRVEILlanCE NEEDS}

This section surveys a sampling of trends in the NAS and the surveillance challenges and requirements they pose. This section examines new entrants in the NAS, space vehicles, Unmanned Aircraft System (UAS) Traffic Management, counter UAS, and security.

\section{New Entrants}

The introduction and integration of new entrants into the national airspace system (NAS) is likely to pose new surveillance requirements. The so-called new entrants, includes a range of non-traditional vehicles and mission types, including unmanned aircraft systems (UASs), and space vehicles. These categories include a wide diversity of sub categories. For example, UASs may fly cargo delivery missions using point-to-point or round robin routes, and UASs may fly agricultural, search-and-rescue, and border patrol missions using orbiting, loitering, and grid patterns.

Some of these mission profiles are currently being flown by manned aircraft, but the number of operations is expected to increase dramatically with UASs due to the lower cost of operations. In other cases, entirely new mission types are likely to emerge. In all cases, UAS performance characteristics are likely to differ from the manned vehicle counterparts (if indeed there is any counterpart). UASs often have lower thrust-to-weight ratios and lower overall performance compared to traditional manned aircraft. But the lack of human occupants and associated supplies could also enable greater thrust-to-weight ratios, and relaxed acceleration constraints. This, along with the tendency toward lower Reynolds Number, especially for small UASs, can lead to faster, more responsive vehicle maneuvers, with tighter turns, with faster initiation and termination rolls, faster accelerations and decelerations, and steeper climb and descent segments. In addition to these performance differences, other UASs have vertical takeoff and landing, as well as hovering, capabilities.

This performance diversity of the new entrants may challenge existing surveillance assets. For instance, ground-based radars use an alpha-beta tracking filter, tuned for conventional aircraft. Furthermore, the new entrants present a wide diversity of operating regimes. They may loiter for days at a time. They may operate at extremely high altitudes, far above conventional aircraft. They may operate at very low altitudes, near or in urban areas and city scape clutter. They may operate near surface terrain and obstacles. All of these conditions are outside the operating regimes that traditional surveillance assets were designed for.

Beyond diverse performance and operating regimes, the new entrants also present diverse cross sections and geometries. UASs can be small, weighing only a few tens of pounds, or less. These small targets, even if within the surveillance asset field of view, may otherwise be undetectable. Other UASs may have several rotors, presenting a non-traditional, dynamic cross section and geometry.

Finally, the entrants present a surveillance challenge simply by virtue of the large numbers, which are anticipated. Currently the number of instrument flight rules flights in the national airspace system per day is counted in the tens of thousands. With the coming new entrants, that number could increase to the hundreds of thousand, or more.

\section{Space Vehicles}

The retirement of the Space Shuttle fleet in 2011 has led to the development of a new generation of commercial space vehicles to lift cargo, supplies, scientific missions, etc., to orbit, and eventually even astronauts to the International Space Station. Examples of the trajectory types of these emerging space vehicles include: vertical takeoff using an expendable launch vehicle, horizontal takeoff and landing using a reusable launch vehicle, air launch from a mothership, vertical takeoff with an RLV where the booster stage is flown back to a designated landing site, and capsule reentry into either sea- or land-based sites. Therefore, as with UASs, space vehicles are expected to use a wide range of trajectories to accomplish their missions. Furthermore, space vehicles operate at a much greater range of speeds, from a standing start at liftoff to significantly higher speeds than conventional aircraft.

At this point in time, however, space vehicle operations in the NAS still occur relatively infrequently and within special activity airspace. Therefore, there has been little in mitigating the impact of these operations on the NAS. But the increase of commercial space vehicle operations, NAS operations and users will be negatively impacted at much higher rates. Delays, reroutes, increased flight time and increased fuel burn will all occur as a result. NAS inefficiencies arise from the need to assume the failure of a space vehicle during launch or reentry. NAS operations and flights must be make highly conservative plans, and avoid the airspace that might be impacted by such failures. With the increase of space vehicle operations, NAS users and service providers will need to develop new procedures, technologies, and infrastructure to adjust and maintain efficiency. One such infrastructure enhancement is improved surveillance to make up for current shortfalls. 


\section{Unmanned Aircraft System Traffic Management (UTM)}

UTM is an Air Navigation Service Provider (ANSP) system that services small UAS operations in otherwise uncontrolled airspace. Small UAS vehicles and missions are diverse, including border surveillance, public safety, agriculture, disaster response, mapping, entertainment and news reporting, cargo delivery, meteorological, and scientific. Most of these diverse missions call for relatively short flights at low altitude, measured in hundreds of feet above ground level. This poses unique surveillance requirements and challenges.

Compared with traditional aviation, small UASs operating at low altitude are impacted more significantly by the wind. Typically, the most important weather phenomenon to the average UAS flight is wind. A strong wind can exceed the small UAS airspeed, so it is unable to return to base. For some small UASs accounting for the wind may be crucial to achieving a successful mission. Key drivers are the wind speed, and the wind spatial and temporal dynamics. Those dynamics are short and fast, respectively, compared to wind patterns above the planetary boundary layer. Furthermore, those dynamics are (i) far more influenced by the local, unique, surface structures and features, and (ii) likely to be chaotic. These factors have implications for the required wind measurement and forecast accuracy. For instance, strong winds or gusty-ness can significantly affect the UAS power budget, as it fights the wind to maintain station. Winds can cut the UAS duration by half. Similarly, the presence of turbulence increases control action, and so reduces battery power. One solution for wind measurement is crowd sourcing the UAS population, though in-situ wind measurement for small UASs can be challenging.

In addition to wind, similar problems exist for other meteorological factors which are important for small UASs, including heavy rain affecting electronics, weight, propeller efficiency, and visibility; temperature affecting batteries (lithium batteries area quite temperature sensitive, and both hot and very cold temperatures cause draw down); thermal effects causing rapid altitude changes of hundreds of feet; lightning causing radio frequency interference, microbursts, and hail.

In meteorological forecasting at low altitudes, the terrain is a dominant factor. There are also seasonal effects, for example, terrain that is lush with vegetation has a different impact on low-altitude weather versus terrain that is dry and brown.

Traditionally in aviation, ceiling and visibility products are associated with an airport. But with UASs they are needed wherever the UAS is operating. Visibility adds a reverse meaning for UASs. In addition to the slant range from the vehicle, visibility is also a measure of the slant range from the ground to the vehicle. In the latter case, one solution for measuring visibility is to exploit the large number of cameras that are common in public places. Image processing techniques would use edge detection of distant objects to infer visibility.
Convection is also a challenge because convection forecast accuracy generally improves with scale. A hurricane is easier to forecast than a $10 \mathrm{~km}$ cell. UASs require small-scale convection forecasts.

In addition to weather, traffic, terrain, and obstacles can also pose surveillance challenges. These challenges include high density traffic, complex (unordered) traffic structure, interaction with manned operations such as crop dusters, small vehicles with low radar and optical cross sections, and so forth. While ground based tracking radars in the airport terminal area provide surveillance tracking data with approximately a 5.5 second period, $t$ the required small UAS tracking surveillance is now considered to be one second.

UASs operating within major cities pose particular surveillance challenges for several different reasons, including highly dynamic winds which can be high speed in given locations, complicated wind modeling and forecasting, vehicle stability, navigation, steering, separation from high value objects such as buildings, surveillance of various types of otherwise unmapped, dangerous objects (power lines, scaffolding, clothes lines, etc.), communications with substantial multipath, and so forth.

As alluded to above, one solution to these various surveillance challenges may be to use information from UAS operations; that is, crowd-sourcing techniques. The UTM concept of operations calls for the sharing of traffic, weather, and terrain information between the UTM and UAS services and the UAS operations. The UTM eco-system facilitates this sharing of information between UAS operations. This paradigm may be critical to address the inherent limitations of surveillance of non-cooperative manned vehicles, low altitude weather forecasts, and vehicle obstruction maps. But this will bring with it many challenges, including:

- Calibration is required to avoid bias errors, but how do you calibrate thousands of sensors distributed on diverse UASs?

- The means for verifying and validating this nontraditional information will have to be established.

- Sensor mounting (UAVs have various shapes and multiple propellers causing unique air flow geometries which may impact the sensor measurements).

- UAVs have limited power and weight restrictions so usually only limited sensors can be accommodated

- Collected data will be very limited in time and space. Should it be used in a synoptic low altitude forecast product? That may be challenging because the observations are clustered in time and space. Or mesoscale or local forecasts could be constructed, specifically for UASs (e.g., forecasts targeted to agricultural areas, power lines, etc.).

- An infrastructure is needed for collecting the observations. 


\section{Counter UAS}

Small UASs are restricted to designated airspaces and pose a surveillance challenge when they depart those regions. Unknown vehicles and their operators are subject to some type of enforcement or immediate mitigation action. For example, small UASs detected near an airport within controlled airspace where its flight is restricted must be detected and intercepted. This likely requires a comprehensive multilayer security approach.

\section{Security}

There are various security threats to aviation in general, and surveillance systems such as ADS-B in particular. Surveillance systems are, in general, cyber-physical systems
(CPSs). In addition to their cyber and physical components, they are the target of both cyber and physical threats. Both types of threats may target both types of components, making for a two-by-two threat matrix.

ADS-B In is particularly vulnerable to these threats. For example, it can be spoofed (fooling receiver with a false signal), and jammed (corruption of a true signal). Developers have proposed solutions for many of its vulnerabilities. For instance, one approach is to use a platoon of nearby flights that transmit ADS-B information between themselves, enabling them to discriminate false ADS-B transmissions. But such solutions represent significant system modifications.

Table 1. Surveillance systems.

\begin{tabular}{|c|c|c|c|c|}
\hline \multirow[t]{2}{*}{ Surveillance Technologies } & \multirow[t]{2}{*}{ Cooperative } & \multirow{2}{*}{$\begin{array}{l}\text { Non- } \\
\text { Cooperative }\end{array}$} & \multicolumn{2}{|c|}{ Targets/Threats } \\
\hline & & & Airborne & Ground \\
\hline \multicolumn{5}{|l|}{ Legacy Ground-installed Surveillance } \\
\hline Airport Surveillance RADARs (ASR's) & & + & + & \\
\hline Air Route Su rveillance RADARs (ARSR's) & + & + & + & \\
\hline Secondary RADARs & + & & & \\
\hline Tethered Aeros tat Radar System (TARS) & & + & & + \\
\hline Terminal Doppler Weather Radar & & + & + & \\
\hline Next Generation RADAR (NEXRAD) & & + & + & \\
\hline Airport Surface Detection Equipment (ASDE/ASSC) & + & + & & + \\
\hline Wide Area Multilateration (WAM) & + & & + & + \\
\hline Inductive Loop Systems & & + & & + \\
\hline \multicolumn{5}{|l|}{ Legacy Aircraft-installed Surveillance } \\
\hline Automatic Dependent Surveillance (ADS-B) & + & & + & + \\
\hline Aire on (Satellite Receivers) & + & & + & + \\
\hline Traffic Collision Avoidance System (TCAS) & + & & + & \\
\hline Air to Air Radars & & + & + & \\
\hline \multicolumn{5}{|l|}{ Future Surveillance Technologies } \\
\hline Ground Based Sense and Avoid (GBSAA) RADARs & & + & + & \\
\hline $\begin{array}{l}\text { Spectrum Efficient National Surveillance RADAR } \\
\text { (SENSR) }\end{array}$ & + & + & + & + \\
\hline LTE Location Based Services (LBS) & + & & + & + \\
\hline Electro Optical (EO) & & + & + & + \\
\hline Infrared (IR) & & + & + & + \\
\hline Millimeter Wave (MMW) & & + & & + \\
\hline LIDAR & & + & & + \\
\hline Real-time Locating Systems (RTLS) & + & & & + \\
\hline Ultrasonic Detection Systems & & + & & + \\
\hline Virtual Radars & + & + & + & + \\
\hline
\end{tabular}

\section{SOURCES OF SURVEILlanCE}

UAS surveillance as considered in this paper includes tracking the location of aircraft in the air and on the ground, and tracking threats to aircraft. Threats to aircraft in the air include other aircraft, weather, birds, ground obstacles, and terrain. Threats to aircraft on the ground include other aircraft, weather, ground obstacles, ground vehicles, people, and animals.
Historically aircraft operators and Air Traffic Control (ATC) have depended upon legacy ground-installed and aircraftinstalled surveillance technologies for safe air traffic operations. These legacy surveillance technologies often support other national needs including border protection. The introduction of UAS operations has created new surveillance challenges related to lower altitude operations, smaller cross section aircraft, and limited avionics SWaP and airborne antennae dimensions. The UAS surveillance challenges have stimulated a search for new aviation surveillance 
technologies to address legacy surveillance technology gaps, and to develop concepts to aggregate and synthesize all available surveillance, to distribute to a broad aviation stakeholder community.

Legacy and future surveillance technologies are summarized in Table 1, and described in more detail in the following sections. Surveillance systems are grouped by legacy groundinstalled surveillance technologies, legacy aircraft installed surveillance technologies, and future surveillance technologies.

The Spectrum Efficient National Surveillance Radar (SENSR) program will replace many of the existing surveillance radar systems with a common system or family of systems, while freeing valuable spectrum for other uses.

Legacy cooperative aircraft-installed systems depend upon position, navigation, and timing (PNT) systems. Legacy PNT systems include GPS, inertial navigations systems, and DME/DME/IRU systems. GPS is considered vulnerable to system loss, jamming, and spoofing. Future PNT systems under consideration by the FAA intended to backup GPS are Enhanced DME (eDME) and a DME Hybrid system which modifies ADS-B RTs to enable 1090/UAT ranging.

\section{Legacy Ground-installed Surveillance Technologies}

Ground-installed RADAR systems. Legacy ground-installed surveillance RADARs include short range primary RADARs associated with Air Traffic Control Towers (ATCT's) and Terminal Radar Approach Control Facilities (TRACON's), long range primary RADARs associated with Air Route Traffic Control Centers (ARTCCs) and border surveillance facilities, secondary RADARs paired with primary RADARs, tethered aerostat RADARs, weather RADARs, and airport surface detection RADARs

Ground-installed radar systems are summarized in the Table 2, and described in more detail in the following sections.

Table 2. Ground-installed surveillance radar systems.

\begin{tabular}{|c|c|c|c|}
\hline Ground installed Surveillance RADARS & FAA & DoD & Range \\
\hline \multicolumn{4}{|l|}{ Legacy } \\
\hline Airport Surveillance RADARs (ASR's) & ASR-7, $8,9,11$ & AN/GPN-12,20,27,30 & $60 \mathrm{~nm}$ \\
\hline Air Route Surveillance RADARs (ARSR's) & ASRSR- $1,2,3,4$ & CARSR FPS- $20,66,67,93$ & $200 \mathrm{~nm}$ \\
\hline Secondary RADARs & \multicolumn{2}{|c|}{ ATCBI- $4 / 5 / 6$, Mode-S } & $200 \mathrm{~nm}$ \\
\hline Tethered Aerostat Radar System (TARS) & L/M L88 & DHS/CBP & $200 \mathrm{~nm}$ \\
\hline Terminal Doppler Weather Radar & TDWR & na & 84 \\
\hline Next Generation RADAR (NEXRAD) & WSR-88D & na & 200 \\
\hline Airport Surface Detection Equipment & ASDE- $3 / X, A S S C$ & na & Airport Surface \\
\hline Future & 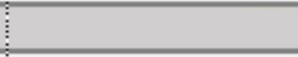 & & \\
\hline $\begin{array}{l}\text { Ground Based Sense and Avoid (GBSAA) } \\
\text { RADARs }\end{array}$ & $\begin{array}{l}\text { Evaluations DFW } \\
\text { /Denver }\end{array}$ & $\begin{array}{l}\text { Air Force eval - ASR-11 (1) } \\
\text { US Army Deploy - LSTAR - } \\
\text { (5) }\end{array}$ & up to $35 \mathrm{~nm}$ \\
\hline $\begin{array}{l}\text { Spectrum Efficient National Surveillance } \\
\text { RADAR (SENSR) }\end{array}$ & Pre-Procurement & Pre-Procurement & $250 \mathrm{~nm}$ \\
\hline
\end{tabular}

Each radar system includes electronic equipment and major infrastructure at the radar antenna site (typically a modular tower, concrete/masonry building, secure perimeter fencing, grounding system, and uninterruptable power system) plus remote electronic equipment at distant operational facilities, including dedicated displays, common workstations, telecommunications and provision for uninterruptable power at the remote sites.

Short range radar surveillance systems. The FAA has developed and deployed an increasing more capable series of short range radars designated as ASR (Airport Surveillance Radars). The military has deployed a derivative series of radars designated by AN/GPN. These radar systems are located on or near major airports and supply primary radar signals in all weather conditions to Air Traffic Control (ATC).

- The current short range radars include the ASR-8 (AN/GPN-20), ASR-9, and ASR-11 (AN/GPN-30).

- The ASR-9 incorporates the Weather Systems Processor (WSP).

- ASR-11 (AN/GPN-30) S-Band and Mobile ASR SBand: is the newest and has replaced several of the radars that were not replaced by an earlier ASR-9 program. The ASR-11 contains its own beacon system, known as the MSSR (Monopulse Secondary Surveillance Radar).

- The Mobile Airport Surveillance Radar (MASR): is a terminal surveillance radar that can be moved from site to site to support radar relocations, temporary planned 
outages of an existing radar for installation of upgrades, and emergency operations when existing systems are damaged. [1]

Long range radar surveillance systems: The FAA has developed and deployed an increasing more capable series of long range radars designated as ARSR (Air Route Surveillance Radars). The military has deployed a derivative series of radars designated by AN/FPS. The ARSRs include weather monitoring.

- The ARSR-4 is the only FAA en route radar with three dimensional (3D) capabilities.

- The military long range radars are modified versions of the same AN/FPS-20 series radar system.

Secondary Surveillance RADAR (SSR) Systems

The purpose of SSR is to improve the ability to detect and identify aircraft while automatically providing the Flight Level (pressure altitude) of an aircraft. An SSR ground station transmits interrogation pulses. An aircraft transponder within line-of-sight range 'listens' for the SSR interrogation signal and transmits a reply that provides aircraft information. The reply sent depends on the interrogation mode. The aircraft is displayed as a tagged icon on the controller's radar screen at the measured bearing and range. An aircraft without an operating transponder still may be observed by primary radar, but would be displayed to the controller without the benefit of SSR derived data.

The FAA has developed and deployed an increasing more capable series of secondary radars designated as ATCBI (Air Traffic Control Beacon Interrogator). The deployed versions today include the ATCBI-4/5/6.

- The ATCBI-6 includes a secure military Identify Friend or Foe (IFF) function that allows it to distinguish between friendly aircraft and enemy aircraft. ATCBI-6 improves system performance through the use of ModeS selective interrogation and monopulse technology. [2]

- Mode-S - The Mode Select mechanism is a ground-based system capable of selective interrogation of Mode $S$ transponders and general interrogation of Air Traffic Control Radar Beacon System (ATCRBS) transponders within range. The system also receives, processes, and forwards the transponder replies to appropriate air traffic control (ATC) automation systems.

\section{Additional Surveillance Technologies}

The following sections summarize additional types of surveillance systems and technologies.

\section{Tethered Aerostat Radar System (TARS)}

Tethered Aerostat Radar Systems are surveillance systems in which the radar is not on the ground, but attached to a tethered helium balloon, shaped like a blimp, that can be stationed above a ground site up to 15,000 feet above the ground, or tethered to a ship. They have been used for 75 years and the most recent use is by Customs and Border Protection along the US southern border for drug interdiction and illegal immigration. TARS radar is the Lockheed Martin L88 wide area surveillance radar with a range of $200 \mathrm{~nm}$. It is used primarily for detecting motion on the surface. Additionally, $449 \mathrm{MHz}$ wind profiler radars have been mounted to aerostats. [3]

\section{Terminal Doppler Weather Radar}

Terminal Doppler Weather Radar (TDWR) is C-Band system used in Air Traffic Control (ATC) to monitor incoming weather and warns air traffic controllers of gust fronts, wind shear and microburst. There are 47 commissioned systems in operation today. The first system was commissioned in 1993.

\section{NEXRAD (WSR-88D)}

The Next Generation Radar (Nexrad) is an S-band, longrange weather radar used by the National Weather Service (NWS) and the FAA. The FAA uses this radar to monitor weather in the en route (long range) environment. There are 155 NEXRAD systems owned by the NWS and 12 FAA offshore systems located in Alaska, Hawaii and the Virgin Islands. The FAA systems are unique by having dual transmitters increasing reliability.

\section{Airport Surface Detection}

Airport surface surveillance systems include the ASDE-X system which is widely deployed today and its replacement, the ASSC system.

- ASDE-X (Airport Surface Detection Equipment Model $\mathrm{X})$ : A runway safety tool providing airport surface surveillance of aircraft and ground vehicles on the movement area for use in air traffic controller displays, especially valuable during darkness and periods of poor visibility. ADSE-X replaces earlier generation surface detection equipment known as ASDE-3 that used primary radar from a tower mounted antenna to detect and display targets on the airport surface. ASDE-X uses transponder returns in addition to primary radar to detect and track targets, providing identification in addition to target location. The ASDE-X interrogators use multilateration that pinpoints the location of the transponder returns by ranging to the aircraft from the multiple locations and solving the geometry where the range arcs intersect. The ASDE-X sites also contain ADS-B receivers to receive these signals from equipped aircraft, and merge the returns to display a single target per aircraft or ground vehicle.

- ASSC (Airport Surface Surveillance Capability): The next generation of surface detection equipment. The ASSC system receives input from ASDE-3 surface movement radar, ASSC multilateration remote units, ADS-B, Airport Surveillance Radar/Mode-S, and Terminal automation for the flight plan data. ASSC fuses this data to produce a highly accurate display for controllers to show aircraft and ground vehicles on the surface movement area, and on arrival and departure 
routes. Pilots with TIS-B and cockpit displays can also receive ASSC data. ASSC data is also transmitted to other FAA systems, including ADS-B, SWIM, and Runway Status Lights. [4]

\section{Multilateration}

Multilateration is the same SSR ranging technique used in ASDE-X, but applied over a wide area instead of just an airport surface. It is used primarily in mountainous areas where radar has difficulty seeing targets behind the mountains. If the target can be seen by enough ground stations, the technique can be used to solve for altitude as well as horizontal position, but the altitude information contained in the aircraft transponder return is used to improve the height resolution. Used widely in Europe, the FAA has only fielded this service in the mountainous area of western Colorado to provide radar services to aircraft using the airports in the ski resort country. This is called the Colorado Wide Area Multilateration (WAM) system.

\section{Inductive Loop Detectors}

The inductive loop detector (ILD) is the most common sensor used in traffic management applications. Its size and shape vary, including the 5 -ft by 5 -ft or $6-\mathrm{ft}$ by 6 - $\mathrm{ft}$ square loops, 6$\mathrm{ft}$ diameter round loops, and rectangular configurations having a $6-\mathrm{ft}$ width and variable length. The principal components of an inductive loop detector include: one or more turns of insulated wire buried in a shallow saw-cut in the roadway, a lead-in cable that runs from a roadside pull box to the controller cabinet, and an electronics unit located in the controller cabinet.

The wire loop is excited with signals whose frequencies range from $10 \mathrm{KHz}$ to $50 \mathrm{KHz}$ and functions as an inductive element in conjunction with the electronics unit. When a vehicle stops on or passes over the loop, the inductance of the loop is decreased. The decreased inductance increases the oscillation frequency and causes the electronics unit to send a pulse to the controller, indicating the presence or passage of a vehicle. [5]

\section{Aircraft-installed Surveillance Technologies}

ADS-B (Automatic Dependent Surveillance). A system which sends aircraft position derived on board to a ground control system for use in Air Traffic Control. ADS-B uses GPS as the position source, broadcasting to ATC ground stations and other nearby aircraft once a second. This broadcast contains the position, altitude, identity and velocity vector of the reporting aircraft. Aircraft broadcast ADS-B messages on the $1090 \mathrm{MHz}$ frequency if above FL 180 and may use the frequency $978 \mathrm{MHz}$, known as the Universal Access Transceiver (UAT), below FL 180.

Aireon. In an effort to support basic radar services worldwide, the Aireon company has put ADS-B transponders on latest Iridium Next satellites in low earth orbit that can receive ADS-B transmissions from aircraft and send them to the appropriate ground ATC facility anywhere in the world.
As oceanic separation standards are much larger than domestic (fifty to one hundred miles vs. three to five miles) the ADS-B messages do not all have to be forwarded, instead only every eight seconds (with a latency of two seconds) to support reducing separation to 10 miles. Also, Aireon's data is encrypted so it is likely to be among the most secure of surveillance sources. Air traffic service providers (ANSPs) in several parts of the world have already signed up to use the service and the FAA has it under consideration for use in the Atlantic and Pacific Flight Information Regions in which they provide the air traffic services.

TCAS (Traffic Collision Avoidance System). Air to air active surveillance of the transponders on other aircraft was developed beginning in the 1970's and formally called for by the FAA administrator in 1981. Standards for the electronics' signal in space and the avoidance logic to ensure cooperative resolutions were developed in RTCA Special Committee 147. Congress required the system by law, followed up by FAA rulemaking that all aircraft in the US with 31 or more seats would have to install and operate TCAS by 1993. Collisions involving equipped aircraft subsequently ceased except for one notable exception in Europe in which the TCAS Resolution Advisory was deliberately ignored by one of the pilots involved in favor of the controller's instructions.

There is a movement to incorporate ADS-B IN surveillance into TCAS to improve upon the accuracy of the encounter geometry available to the avoidance logic. There are also many who believe that large UAs should carry TCAS even though they would not be required to under the seat rule.

Air to Air radars. Historically, air to air radar has been a special purpose tool largely limited to military operations. It has been very expensive, heavy, with limited performance and high-power requirements. Most airborne radars do not scan completely around the aircraft, rather in a fairly narrow field of view to the front. The requirement for UAs to perform an equivalent "see and avoid" function to the manned aircraft requirement brought renewed interest in the use of airborne radar for surveillance of non-cooperative targets.

In the past, aircraft-installed C-Band weather radars were quite adept at picking up other aircraft in their scan. Because non-cooperative targets don't report their altitude, this sensitive parameter must be gleaned from the elevation angle of the return and the range to the target, so the local horizontal must be accurately known even during maneuvering. One idea being examined is to integrate airborne radar with an electro-optical/IR sensor. The radar is very accurate in range but not in azimuth. Electro-optical sensors (cameras) are the opposite, accurate in azimuth but no clue about range without time consuming tracking and processing. Integrating the two concepts might produce a viable surveillance device at least for use in good visibility conditions

\section{Future Surveillance Technologies}

Ground Based Sense and Avoid (GBSAA) RADARs. A number of concepts are being explored for non-cooperative 
detection of UAS. The FAA and the Department of Homeland Security (DHS) conducted drone-detection research in the vicinity of Denver International Airport. This work is part of the FAA's Pathfinder Program for UAS Detection at Airports and Critical Infrastructure. The work in Denver is one of six technical evaluations. Industry partners involved in the Denver flights included CACI International, Liteye Systems and Sensofusion. Other evaluation sites include Atlantic City International Airport, JFK International Airport, Eglin Air Force Base, Helsinki Airport, and DallasFt. Worth International Airport. In addition to DHS, the FAA's federal research partners include the Department of Defense, FBI, Federal Communications Commission, Department of the Interior, Department of Energy, NASA, Department of Justice, Bureau of Prisons, US Secret Service and US. [6]

SSRC has produced a family of radar systems designed for GBSAA named "LSTAR" ground sensors. The LSTAR is certified to DO-178 standards, produces 360-degree coverage with a range of $50 \mathrm{KM}$ and is advertised to detect low reflectivity targets like hang gliders. It is "small, transportable, can cue a visible light or IR camera and can be integrated with ADS-B or SSR data." The systems are being evaluated at Dallas Fort Worth (DFW) by the FAA.

In a system test run at Cannon Air Force Base in New Mexico, a modified STARS automation system was used, including primary radar data to track the non-cooperative targets. [7] The U.S. Army installed its first Ground-Based Sense-and-Avoid radar system at Fort Hood, which is home to two MQ-1C Gray Eagle unmanned aircraft system companies, in December 2014. Fort Hood is one of five installations that have been identified to acquire the system. [8]

Spectrum Efficient National Surveillance RADAR (SENSR) Program. SENSR is currently slated to replace many, if not all, distinct site configurations of legacy surveillance systems.

A DoD, DHS, DOT/FAA, DOC/NWS (NOAA) cross-agency program titled Spectrum Efficient National Surveillance Radar (SENSR) has been initiated and is seeking to make available a minimum of $30 \mathrm{MHz}$ in the $1300-1350 \mathrm{MHz}$ band for reallocation to shared Federal and non-Federal use by updating some or all of the legacy surveillance technologies. This multi- agency program led by the FAA is specifically targeted at vacating federally-used spectrum in the L-band to make available that $30 \mathrm{MHz}$ for auction to commercial spectrum users. This auction is estimated to produce nearly $\$ 50 \mathrm{Billion}$ in revenue to the federal government and is slated to reimburse the cost of updating and relocating legacy systems in that band to function in another available spectrum as well as update/modernize aging cooperative and non-cooperative surveillance systems supporting air traffic control, homeland security, air defense, and weather prediction capabilities required by key U.S. Federal agencies
LTE location systems. LTE has Location Based Services (LBS) written into the LTE specification. The services are rapidly improving with each generation of LTE technology. The benefit of LTE LBS is to supplement GPS in environments where obstructions interfere with GPS signals, e.g. urban environments. LTE LBS allow surveillance of all LTE enabled entities. The LTE LBS infrastructure is already in place and is expanding and improving continuously as LTE providers invest in their networks and network services. [9]

Location Based Services (LBS) is already well established, using the location of the mobile device for both emergency services (E911,) and infotainment (map services, directions to a chosen location, local advertising/information and "find a friend"). So far, this is just the beginning for LBS; the increasing sophistication of the smart phone, high-speed data rates with LTE, and consumer demand for 'always-on' interaction mean that LBS applications are going to expand massively over the coming years.

Electro Optical systems. Electro optical systems (cameras) are a common sensor on UAS and are an evolving option for both UAS detect and avoid (DAA) and for navigation during day light hours. The DAA application, like air to air radars, can notionally detect and monitor non-cooperative vehicles within EO range of the EO sensor, acting as pilot eyes in the UAS. Range is a function of EO optics, atmospheric conditions, and lighting. EO sensors are passive, i.e. they do not transmit, so spectrum use is not an issue.

Infrared (IR) systems. Infrared systems (thermal cameras) are a common sensor on UAS and are an evolving option for both UAS detect and avoid (DAA) and for navigation during night time hours. The DAA application, like air to air radars, can notionally detect and monitor non-cooperative vehicles within IR range of the IR sensor. Range is a function of IR optics, atmospheric conditions, and target heat signature. IR sensors are passive, i.e., they do not transmit, so spectrum use is not an issue.

Millimeter Wave (MM). Millimeter Wave (MMW) radars are currently used as range measuring devices in applications such as automotive driving aids, the mapping of mines, and autonomous field robotics. This recent interest is largely due to the advantages MMW radars offer over other range measuring sensors, as their performance is less affected by dust, fog, rain or snow and ambient lighting conditions. MMW radars can provide received signal strength values, at all discrete range intervals, within the working range of the radar. The received power versus range spectra hence contain useful range to target information, but are also corrupted by noise. User defined stochastic algorithms can then be implemented, which exploit this rich data to improve object detection and mapping performance. This is in contrast to many other range measuring devices which typically internally threshold received signals, to provide single hard decisions only, on the estimated range to objects. 
Light Detection and Ranging (LIDAR). LIDAR iIs a remote sensing method that uses light in the form of a pulsed laser to measure ranges (variable distances) to the earth. These light pulses - combined with other data recorded by the airborne system - generate precise, three-dimensional information about the shape of the Earth and its surface characteristics. [11]

A LIDAR instrument principally consists of a laser, a scanner, and a specialized GPS receiver. Airplanes and helicopters are the most commonly used platforms for acquiring LIDAR data over broad areas.

Most self-driving vehicles use LIDAR to map physical space by bouncing laser beams off of objects. But as we've reported recently, the autonomy boom means that suppliers of the once-niche hardware are struggling to keep up with demand. Even companies that have developed in-house alternatives are having trouble: Uber and Waymo are currently embroiled in a lawsuit over the intellectual property relating to their homegrown hardware. [12]

Typically, a LIDAR rig is the most distinctive part of a selfdriving car: it looks like an oversize coffee can mounted on the car's roof, whirling around as it spits out laser pulses. And the most commonly spotted sensors are made by Velodyne, whose top-end devices cost tens of thousands of dollars.

Real-time locating systems (RTLS). Real-time locating systems (RTLS) are used to automatically identify and track the location of objects or people in real time, usually within a building or other contained area. Wireless RTLS tags are attached to objects or worn by people, and in most RTLS, fixed reference points receive wireless signals from tags to determine their location. Examples of real-time locating systems include tracking automobiles through an assembly line, locating pallets of merchandise in a warehouse, or finding medical equipment in a hospital.

The physical layer of RTLS technology is usually some form of radio frequency (RF) communication, but some systems use optical (usually infrared) or acoustic (usually ultrasound) technology instead of or in addition to RF. Tags and fixed reference points can be transmitters, receivers, or both, resulting in numerous possible technology combinations.

RTLS are a form of local positioning system, and do not usually refer to GPS or to mobile phone tracking. Location information usually does not include speed, direction, or spatial orientation.

Ultrasonic detection systems. Ultrasonic sensors transmit pressure waves of sound energy at a frequency between 25 and $50 \mathrm{KHz}$, which are above the human audible range. Most ultrasonic sensors operate with pulse waveforms and provide vehicle count, presence, and occupancy information. Pulse waveforms measure distances to the road surface and vehicle surface by detecting the portion of the transmitted energy that is reflected towards the sensor from an area defined by the transmitter s beam width. When a distance other than that to the background road surface is measured, the sensor interprets that measurement as the presence of a vehicle. The received ultrasonic energy is converted into electrical energy that is analyzed by signal processing electronics that is either collocated with the transducer or placed in a roadside controller.

Virtual Radars. Encompasses a concept to collect, aggregate, and synthesize ground-installed and aircraft-installed surveillance and DAA information, to distribute to UAS operators, ATC, and the broader aviation community. The concept also envisions crowd sourcing UA DAA data, UA weather data, non-aviation security data, and non-aviation weather data (especially applicable to micro weather).

\section{Architectural Alternatives}

The system architecture is designed to accomplish the following objectives:

- Collect and "Fuse" aircraft data from many different data sources.

- Collect airspace data from various sources.

- Detect aircraft-to-aircraft conflicts and/or anomalous behavior.

- Detect aircraft-to-environmental conflicts.

- Provide service users with several avenues for receiving and viewing the aircraft data and/or alerts.

The system is comprised of several components which, working together, accomplish the above stated objectives, see Figure 1. The system is flexible enough to be located at a single facility, hosted in the cloud, or to be distributed between several sites, including a cloud hosting service. 


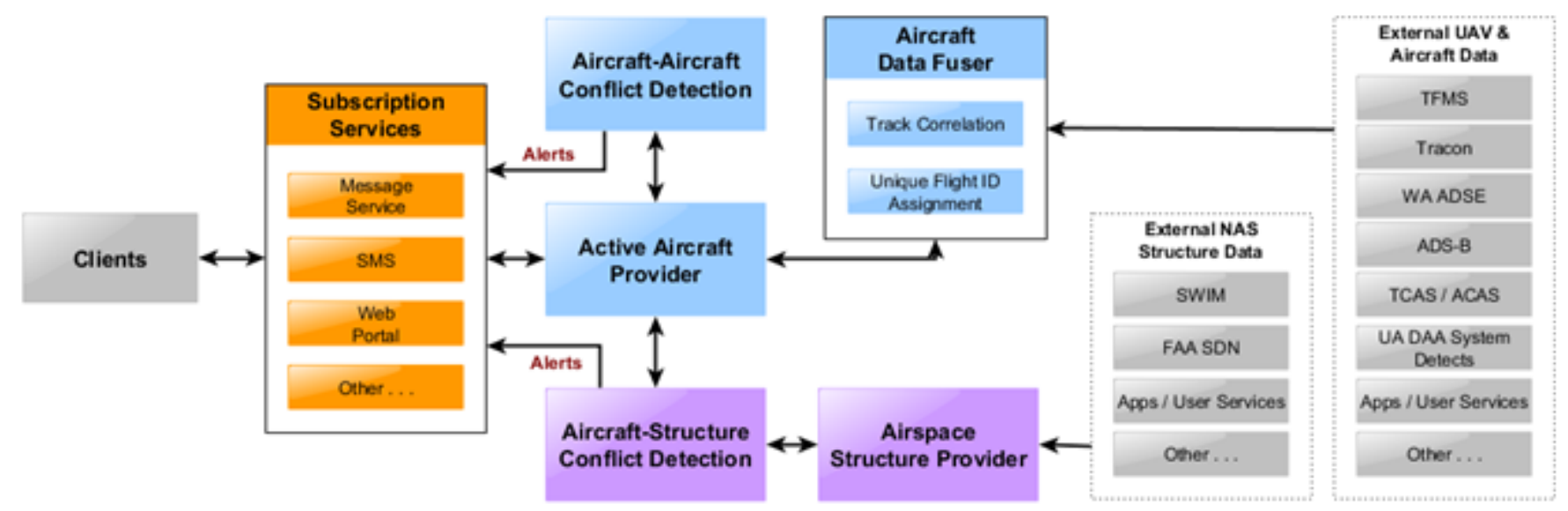

Figure 1. System Architecture

\section{Aircraft Data Fusing and Conflict Detection}

The first three sets of components (colored in blue in the System Architecture diagram) are responsible for handling the processing the aircraft data. These components are the Aircraft Data Fuser, the Active Aircraft Provider, and the Aircraft-to-Aircraft Conflict Detector.

The Aircraft Data Fuser (ADF) collects data from many different sources (see External UAV Aircraft Data sources colored in gray in the System Architecture diagram) and fuses/merges all of this data into unique aircraft objects. Because aircraft IDs, if even available from the data source, will be different between the sources. Therefore, it is the ADF's job to correlate the various aircraft reports using all of the available data (geographic, time, aircraft type, performance, etc.) and to then provide the rest of the system with a global unique flight id (GUFI) for each flight. The uniquely identified flights and information are then passed onto the Active Aircraft Provider.

The Active Aircraft Provider (AAP) receives flight records from the ADF, updates the flight state of its internal list of flights, and provides an update notice to any components listening for flight updates: the Aircraft-to-Aircraft Conflict Detection component, the Aircraft-to-Structure Conflict Detection component, and the Subscription Services component.

The Aircraft-to-Aircraft Conflict Detection (AADC) component performs conflict detection calculations whenever it receives flight updates from the AAP. It utilizes the current aircraft position reports, estimates of direction and speed, and updates to internal models to determine if any aircraft will be entering a conflict state with other aircraft, and if so, to flag the aircraft and send out alerts to the Subscription Services. Additionally, the AADC can examine an aircraft's state against planned routes (if available) and provide notifications and/or alerts to the Subscription Service for aircraft deviations from its plan.
Airspace Structure Data and Conflict Detection

The second set of components (colored in purple in the System Architecture Diagram) are responsible for processing environmental and airspace data and in making sure that aircraft are in compliance with any regulations, restrictions, or safety factors expressed by the airspace data.

The Airspace Structure Provider (ASP) collects airspace and environmental data from various data sources (see External NAS Structure Data in gray in the System Architecture diagram), performs any needed pre or post data processing on the data, and then stores/updates the airspace structure data in its internal list of active (or soon to be active) structures. After any new additions or updates, the component updates any other listener components of the changes: namely the Aircraft-to-Structure Conflict Detector.

The Aircraft-to-Structure Conflict Detector (ASCD) performs a job similar to the Aircraft-to-Aircraft detector; however, it is interested in making sure that aircraft are in full compliance of any airspace restrictions or regulations through which they are traversing. If any aircraft is found to be non-compliant, that flight is flagged and an alert is sent out to the Subscription Service.

\section{Subscription Services}

The third and last set of components is the Subscription Services component. This component receives aircraft information from the Active Aircraft Provider and alerts and notifications from the Aircraft-to-Aircraft and Aircraft-to-Structure Conflict Detectors. The job of this component is to provide this aircraft data and information to external clients through multiple portals. Simple portals consist of short SMS messages or e-mail updated to notify clients of any important updates or alerts. A more advanced portal consists of a virtual radar covering regions of the NAS that can be viewed from a web browser, allowing clients to see not only their aircraft, but other aircraft and airspace structure that are active in their area of operation. 


\section{UAS Integration into Terminal areas with GBSAA}

The proliferation of unmanned aircraft (UAS) into the NAS has raised safety concerns for the FAA. UAS introduction increases the number of vehicle in the airspace. The UAS when compared to manned aircraft are often small and hard to 'see,' possess different performance characteristics, often fly unique mission profiles, carry fewer and less capable avionics and sensors for maintaining safe separation, and operate out-of-sight of the UAS operator who lacks an on the aircraft "see and avoid" capability.

Federal regulation requires pilots to be aware of all other surrounding aircraft - either visually or using on-board instrumentation-and to safely avoid near-misses or collisions. Because there is no pilot physically onboard a UAS, remote operators must resort to limiting and sometimes impractical means of seeing and avoiding other aircraft, such as ground-based observers or chase aircraft behind the UAS.

The aviation community has defined a limited scope Detect and Avoid (DAA) approach described by the RTCA SC228 DAA MOPS. However, the scope of the current RTCA DAA MOPS does not include operations in terminal areas.

In today's busier terminal areas, manned aircraft separation is achieved by ATC use of radar systems such as the ASR9 / ASR-11, ADS-B, and pilot "see and avoid" behaviors. The ASR radar systems are typically tuned to the faster and larger manned aircraft and cannot reliably detect UAS.

To provide ATC and UAS operators see and avoid capabilities in the terminal area, the Air Force, the MITRE Corporation, Raytheon, the Volpe Center, members of the FAA, and many others have been developing a Ground Based Detect and Avoid (GBDAA) capability. The Air Force solution processes and fuses radar data from ASR terminal radars and from local GBSAA radars tuned to capture slow and small targets into a display that can provide ATC and UAS operators a detailed view of UAS and manned aircraft operations in the terminal area.

The Air Force GBSAA solution is operational today at Cannon Air Force Base where it fulfills the FAA's See and Avoid requirement (14CFR\$91.113). The GBDAA system is designed to utilize existing ground-based radar infrastructures (ASR-9 and ASR-11) that work together with other small target ground-based radars (STARS, LSTAR, Sentinel) to provide operational personnel with situational awareness of the terminal airspace. The Airport Surveillance Radar (ASR) system allows operators to detect positions of cooperative aircraft, while the Standard Terminal Automation Replacement System (STARS), LSTAR, and Sentinel systems allow operators to filter out the airspace and detect non-cooperative aircraft. The position and movement of aircraft is indicated on a display, centered on the UAS, that a UAS operator can use to facilitate self-separation. This system implements a collision detection algorithm to alert an operator to an impending collision with another aircraft. Audible and visual alerts are presented to a UAS operator when a threat aircraft enters a pre-defined area around the UAS. If evasive action is required, additional alerts are provided to the pilot.

The Cannon Air Force GBSAA system has obtained both an FAA Certificate of Authorization (COA) and an Airworthiness Military Flight Release (MFR) that allows UAS to transit to RAs through the NAS. From this certification case, many lessons were learned as to how to create and gain the rigorous approval from both the FAA and the Air Force. Since then, additional Air Force bases have initiated the process for establishing GBSAA systems.

As shown in Figure 2, multiple flight operations are being performed. The GBDAA STARS screen is utilizing the fused data from the ASR-11, as well as its own algorithm sensors to provide a feed of all the aircraft flying in the terminal area. Before GBDAA, UAS missions would need to put up a Temporary Flight Restriction (TFR), in the terminal area, so they could take off without interruptions from other aircraft. Now, GBDAA system provides the UAS crew with surrounding aircraft positioning information so it can take evasive action, when necessary. It is then an easy step to envision that the same GBDAA produced aircraft positioning tracks being fed into a shared distribution service. In a sense, this would provide a virtual radar display for any UAS crew, in the operational area, to use as a means of detect.

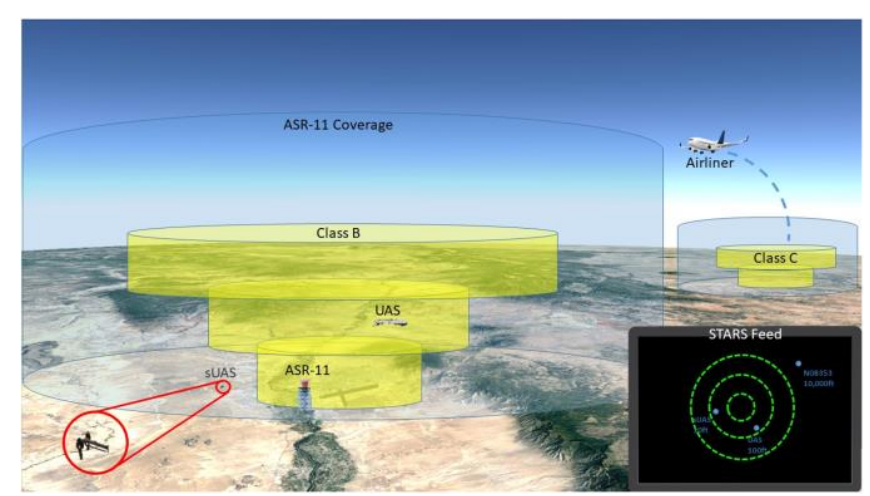

Figure 2. GBDAA concept to support terminal area UAS DAA.

In the future, the fusion of a broad range of surveillance sources will create a Virtual Radar picture for situational awareness, enhancing both air and surface DAA, and enabling terminal see and avoid capabilities superior to that of manned aircraft. Figure 3 depicts a notional simplified view of surveillance sources and the consumers of the Virtual Radar situational awareness data. Various levels of processing may occur at the surveillance sources, the Virtual Radar, and by the consumers of the situational awareness data. ATC will have direct access to the NAS Systems and NAS Services, however the Virtual Radar inclusion of other sources may prove valuable to ATC. 
Aviation stakeholders include, for example, facility managers requiring awareness of non-cooperative aircraft. The Virtual Radar system could be managed by a federal agency or by services created by one or more commercial enterprises.

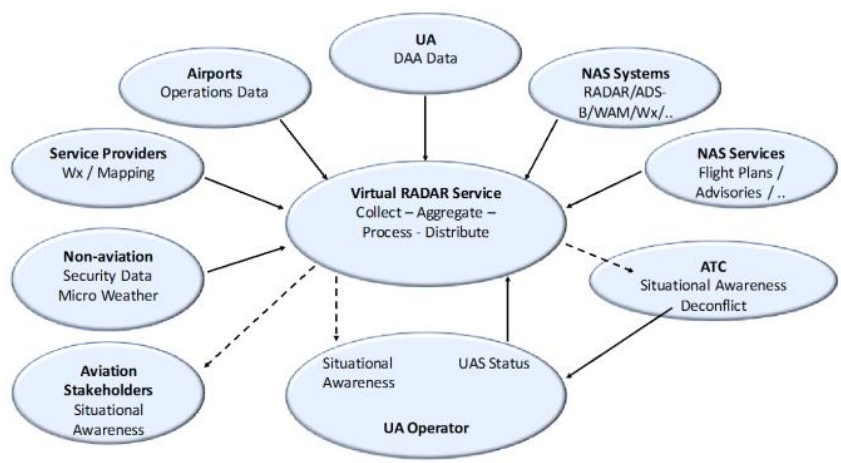

Figure 3. A notional view of virtual radar sources and consumers of the situational awareness data.

\section{Conclusions}

This paper has reviewed the future surveillance needs in the NAS, the diverse surveillance assets in the NAS, and candidate system architectural alternatives for using those surveillance assets to meet future needs. While investment and planning will be required to meet those needs, current and emerging surveillance technologies appear to be sufficient to meet those needs.

\section{ACKNOWLEDGEMENTS}

The authors offer their thanks to the U.S. Department of Transportation, the U.S. Department of Defense, and the U.S Air Force for their support in preparing this paper.

\section{DisCLAIMER}

The text and graphics within this paper express only the views and/or opinions of the authors as individuals and do not represent endorsements, commitments or polices of their firm, agency or organization.

\section{REFERENCES}

[1] FY 2018 FAA Capital Investment Plan, p. 45.

[2] Regulus-group.com, 10-14-2017.

[3] Tethered Aerostat Radar System, fas.org, (Federation of American Scientist), 10-14-2017.
[4] “ADS-B / Airport Surface Surveillance Capability (ASSC)," faa.gov, 10-14-2017.

[5] Surface Surveillance Industry Day Overview, FAA, June 15, 2017.

[6] "FAA Evaluates Drone Detection Systems Around Denver," faa.gov, November 2016.

[7] 'USAF validates Raytheon's GBSAA radar capabilities," airforce-technology.com, November 2012.

[8] "Army installs first Ground-Based Sense-and-Avoid radar," army.mil, PEO Aviation, December 2014.

[9] Mike Thorpe, M. Kottkamp, A. Rössler, J. Schütz., "LTE Location Based Services Technology Introduction White paper," Rohde \& Schwarz, April 2013.

[10] Jose Ebi, Martin Adams, John Stephen Mullane, Nicholas M. Patrikalakis. "Predicting Millimeter Wave Radar Spectra for Autonomous Navigation," IEEE Sensors Journal 10, no. 5 (May 2010): 960-971.

[11] "What is LIDAR?," NOAA, oceanservice.noaa.gov, august 17, 2017.

[12] Jamie Condliffe, “A Key Piece of Self-Driving Cars Is About to Get a Lot Cheaper," MIT Technology Review, April 20, 2017.

\section{BIOGRAPHY}

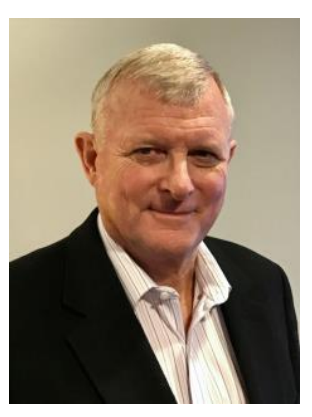

Terry Blumer is an associate with Aviation Management Associates (AMA), a firm helping aviation industry partners and government agencies address air traffic control challenges for over 35 years. $\mathrm{Mr}$. Blumer specializes in unmanned systems and air traffic control issues. Mr. Blumer led unmanned system applications and managed the FAA NAS Voice System program for Harris, headed Business Development and directed the FAA Enhanced Tower Voice Switch program for Denro, and ran the commercial avionics product line management organization for Bendix. Mr. Blumer earned an MBA from the Stanford Graduate School of Business and technical degrees from MIT's Flight Transportation Laboratory in the Department of Aeronautics and Astronautics. 


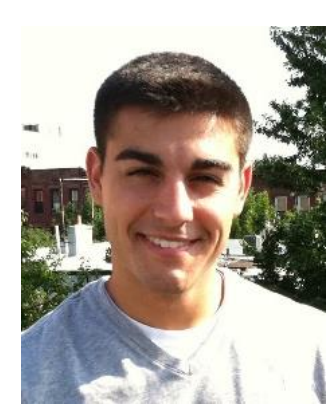

Pete Carros is a Sr. Analyst and System Safety Engineer for Mosaic ATM, Inc. - a firm specializing in air traffic management systems development, unmanned systems and data management systems for the aviation sector. He works under the Autonomous Systems Group and supports the Air Force, through Volpe, on the Ground Based Detect and Avoid (GBDAA) contract. He has assisted the Air Force Safety Center's Remotely Piloted Aircraft (RPA) Brach, as a Systems Safety Engineer. His prior roles include providing safety engineering analysis on DoD Airworthiness projects, safety oversight of Ground Base Sense and Avoid (GBSAA) efforts, assessment and mitigation of GBDAA hazards, and assist with investigations of RPA mishaps. He has a BSME from the Embry Riddle Aeronautical University and is working on a Safety Certificate from the University of Southern California. He has completed the Chief of Safety and Board President Course from the Air Force Safety Center.

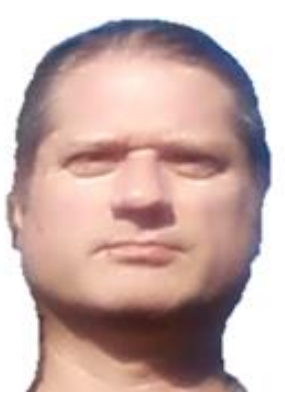

John DiFelici is a principle software engineer for Mosaic ATM, Inc. developing unmanned software systems for the aviation sector. He has worked for over 20 years developing air traffic management, environmental management, and data management systems for the aviation sector. He received a B.S. in Mathematics and Physics from Santa Clara University and his Masters of Physics from the University of Maryland, College Park.

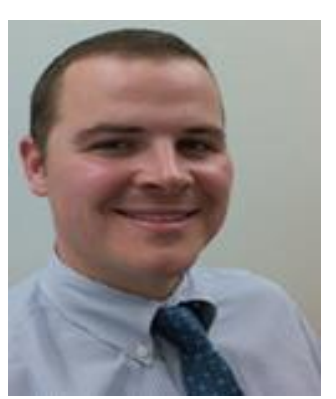

Jason Glaneuski is a Division Chief in the Air Traffic Management Systems Division at the Volpe National Transportation Systems Center (Volpe Center) in Cambridge, MA. His Division applies information technology and operations research disciplines to enhance the capacity, safety, and security of the National Airspace System. A key component of this work is developing concepts and designing automated decision-support tools and capabilities that provide solutions to existing and anticipated traffic flow issues. Mr. Glaneuski has experience both performing and managing technical work in the areas of traffic flow management (TFM), time-based flow management (TBFM), and unmanned aircraft systems (UAS) sense and avoid (SAA), among others. Mr. Glaneuski is a graduate of Daniel Webster College

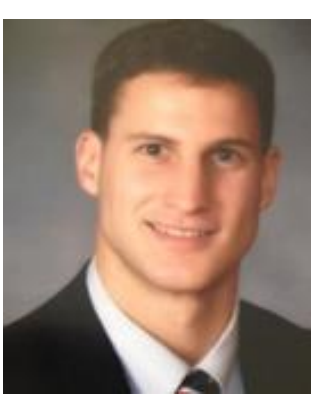

Dylan Hasson is an Operations Research Analyst in the Air Traffic Management Systems Division at the Volpe National Transportation Systems Center (Volpe Center) in Cambridge, MA. The ATMS division blends air traffic operations research, information technology, computer science, and engineering expertise focused on developing and deploying systems that help the air transportation enterprise to operate safely and efficiently. Mr. Hasson is a graduate of Rensselaer Polytechnic Institute where he received a B.S. in Aeronautical Engineering. He is also a private pilot.

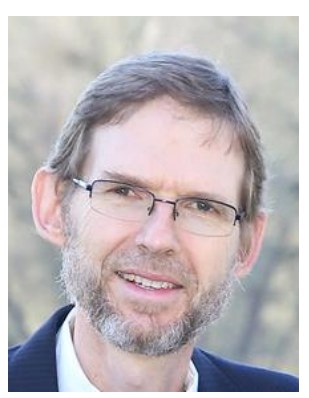

George Hunter is a principle analyst for Mosaic ATM, Inc. - a firm specializing in air traffic management systems development, unmanned systems and data management systems for the aviation sector. He has 30 years of research and development experience in science and engineering. For more than 20 years Dr. Hunter has conceived, performed and directed extensive research studies. He is a graduate of the University of Illinois where he received a Ph.D. in Biophysics. He also received a B.S. and M.S. in Aerospace Engineering from the University of Michigan

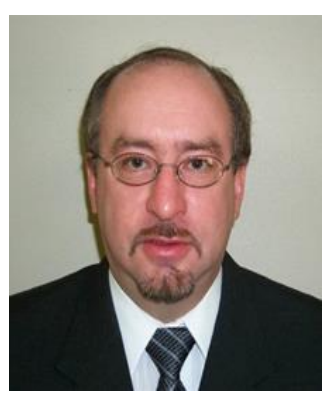

Robert J. Kerczewski has been involved with research and development of satellite and aeronautical communications systems and applications for the Analex Corporation (1982-1986) and NASA (1986-present). He holds a BEE degree from Cleveland State University (1982) and an MSEE degree from Case Western Reserve University (1987). He is currently the Spectrum Element Manager for the NASA's Unmanned Aircraft Systems Integration in the National Airspace System (UAS in the NAS) Communications Sub Project.

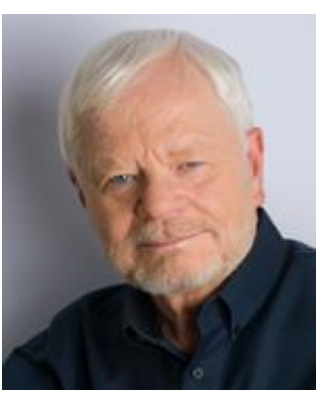

Chris Wargo is a program manager and director business development for Mosaic ATM, Inc. - a firm specializing in air traffic management systems development, unmanned systems and data management systems for the aviation sector. He also leads the Autonomous Systems Group and served as a Chair of the System Engineering Working Group of RTCA SC-203. He has also held positions as President of Computer Network 
\& Software, Inc., Vice President and General Manager for ARINC, Inc., C3I Program Manager RCA Automated Systems and GE, as well as Systems Engineer for GTE Sylvania, Electronic Systems Group, and the US Army. In his role as a leader in aviation next generation systems engineering, he has participated in numerous ICAO, RTCA, AEEC, IEEE and IATA committees and standards working groups throughout his 30 year defense and aeronautical systems career. He has presented a number of papers and chaired many industry conference sessions related to the CNS and network system programs, project and technologies of the general, business and air transport community. He has a BSEE from the University of Wisconsin and an MS, Systems Engineering, from the University of Southern California, and has attended the Defense Systems Management College and the Advanced Management Program at the Harvard Business School 